gr-qc/0012049

Nature 408 (2000) 661-664

\title{
Quantum theory's last challenge
}

\author{
Giovanni AMELINO-CAMELIA \\ Dipartimento di Fisica, Università "La Sapienza", P.le Moro 2, I-00185 Roma, Italy
}

\begin{abstract}
Quantum mechanics is now 100 years old and still going strong. Combining general relativity with quantum mechanics is the last hurdle to be overcome in the "quantum revolution".
\end{abstract}

\footnotetext{
${ }^{1}$ This is the preprint version of an article that appeared in the issue 6813 (volume 408) of Nature, as part of a 3-article celebration of the 100th anniversary of Planck's solution of the black-body-radiation problem. The text of the published version is not exactly the same; moreover, the published version contains 4 illustrations, which are not included here. The article is intended for a wide non-technical readership, but it is not really self-contained, since it relies on points discussed by Anton Zeilinger in another article composing the celebration.
} 
This year we celebrate 100 years of quantum theory, and in particular the anniversary of an announcement made by Max Planck at a meeting of the German Physical Society on 14 December 1900. Planck was interested in the nature of radiation emitted by hot objects, and in 1900 he devised a theory that described all of the experimental evidence, but that required a radical new concept: energy is not emitted or absorbed continuously, but in discrete amounts, called quanta. At the time, Planck was not aware of the profound consequences of his work, but gradually physicists realized that they needed quantum concepts to understand the structure of all matter and radiation.

Over the past century there have been many successful tests of quantum mechanics (see accompanying commentary by Anton Zeilinger in this issue [1]). Experiments have confirmed even some of the most counterintuitive predictions of quantum theory, including "particle-wave duality", the idea that a particle should be treated as a wave. But some physicists still wonder whether quantum theory is a truly fundamental ingredient of the laws of nature, or just a convenient description of some aspects of the microscopic world. It is still possible that quantum mechanics is an approximation to a more fundamental theory, just as Newtonian gravity is a special case of the more accurate description of gravity and the relationship between space and time provided by Einstein's general relativity theory.

The biggest challenge to accepting quantum mechanics as a fundamental theory of nature is that despite 70 years of attempts [2] it has still not been integrated with the classical theory of general relativity. Most of the time, the subtleties of quantum mechanics can be safely ignored by general relativity: gravity drives the expansion of the Universe and the formation of galaxies, whereas quantum theory reigns supreme at the atomic scale. But there are times when quantum mechanics cannot be excluded. For example, a theory unifying gravity and quantum mechanics is required to understand the "Big Bang" - the first few moments of the universe when gravitational interactions were very strong and the scales involved were all microscopic.

Another area of concern is the lack of experimental evidence on the interplay between quantum theory and general relativity. The structure of the two theories does allow for situations in which neither can be neglected, but it is extremely hard to create the required conditions in a laboratory. This is crucial if we are to test any of the theoretical ideas that propose to unify quantum mechanics and general relativity.

\section{The incomplete revolution.}

The "relativity revolution" and the "quantum revolution" are among the greatest successes of twentieth century physics, yet the theories they produced appear to be fundamentally incompatible. General relativity remains a purely classical theory: it describes the geometry of space and time as smooth and continuous whereas quantum mechanics divides everything into discrete chunks. The predictions of the two theories have been confirmed in a large number of experiments, but each of these experiments is relevant for only one or the other of the two theories because of the different scales involved. Physicists' discomfort with this situation is perhaps best described by Rovelli's characterization [3] of the 1900s as "the century of the incomplete revolution", suggesting there may be a greater revolution to come.

Part of the underlying theoretical incompatibility arises from the way the two the- 
ories treat the geometry of space and time. In quantum mechanics, space-time has the role of a fixed arena within which one describes the evolution of various "quantum observables", such as the position of particles. But in general relativity, space and time are dynamical quantities that can respond to and influence other physical processes, such as the movement of planets. So, in principle, the particles in a quantum mechanics experiment affect the evolution of space-time through the gravitational fields generated by their energies. In practice, the particles are usually so small that they have a negligible effect on space-time dynamics, and general relativity can be ignored. This has been the case in all successful test of quantum mechanics.

\section{Approaches to quantum gravity.}

The search for a more fundamental theory, in which the incompatibility between general relativity and quantum mechanics be resolved, can take two different paths. The most drastic option is to look for an alternative theory based on principles that are profoundly different from the principles of quantum mechanics. In such a theory quantum mechanics would only be meaningful as an approximation [4, 5]. The other option is to maintain the existing principles of quantum mechanics but develop a more fundamental theory that can answer questions of a different type [3], such as the ones emerging with a dynamical space-time. The situation in which quantum mechanics is abandoned at the level of the more fundamental theory is a much harder challenge, because one would need to rewrite the rulebook, and no serious candidate has yet emerged. The second option is more easily explored: at least one has quantum theory as a starting point.

There are currently two mature theories based on quantum mechanics that attempt to unify general relativity and quantum mechanics: "canonical quantum gravity" [3, 6] and "superstring theory" [7, 8]. Although they share a common goal these two theories are quite different in the way they approach the technical and physical problems that emerge when building a quantum picture of gravity and space-time. In particular, they differ in the way they handle the mathematical infinities that naturally occur in quantum descriptions of gravitational fields. These infinities are not peculiar to quantum gravity: for example, they also appear in the quantum description of electromagnetic fields (the subject of quantum electrodynamics or QED) but in QED they can be removed by a technique known as perturbative renormalization. Thanks to this technique, QED has become one of the most accurate and best-tested theories of modern physics. But perturbative renormalization does not work for the quantization of Einstein's theory of gravity: the mathematical infinities are too persistent. In canonical quantum gravity "non-perturbative renormalization" is being tried. In "superstring theory" perturbative renormalization can be made to work if it is assumed that the particles we perceive (with the relatively low resolution we presently have) as point-like are actually more properly described using one-dimensional entities (strings) that exist in a space-time with ten dimensions. These ten dimensions include the four we ordinarily perceive (three of space and one of time) plus six other dimensions that would only show up in experiments at extremely small sales. None of these extra dimensions, or any of the string-like states, have yet been observed.

The two approaches also differ in the way they deal with the most fundamental 
challenge to the unification of general relativity and quantum mechanics: replacing the fixed space-time arena with a dynamical version. In "superstring theory" the idea of a fixed space-time arena is conserved, but the theory includes some dynamical variables that also describe space-time. The end result is a dynamical space-time (which, in a sense, is the "sum" of the classical fiducial starting point and some dynamical quantum space-time effects), but some physicists feel that the starting point provided by the fiducial space-time arena does not fully embrace the lessons taught by general relativity. In this respect "canonical quantum gravity" is more ambitious: space-time is fully dynamical from the start. Unfortunately it is not yet clear how this quantum dynamical space-time will describe the important limiting cases (corresponding to the majority of the viable experimental situations) where space-time does behave as a classical and fixed arena.

\section{A quantum theory of space-time.}

One of the most exciting possibilities emerging from "canonical quantum gravity", "superstring theory" and other (less developed) approaches to the unification of general relativity and quantum mechanics is the idea of a space-time that is itself quantized. This could involve a discretization of space-time and/or a quantum "uncertainty principle" similar to that found in ordinary quantum mechanics. In a space-time with a quantum uncertainty principle it would not be possible to measure accurately the distance between two space-time points, just as in ordinary quantum mechanics it is not possible to measure (simultaneously) the position and momentum of a particle within the classical space-time arena.

The quantum description of space-time would require a profound renewal of fundamental physics. For example, it might have unexpected effects on the propagation of particles. These effects would only emerge at the small scale of the Planck length (which is not easily explored experimentally). A useful analogy is the one of the surface of a wooden table. We usually perceive the surface as perfectly flat, but if we look at the table with a microscope (which allows us to acquire sensitivity to its short-distance structure) it becomes clear that it is not exactly flat. The flatness we ordinarily perceive is some sort of averaging over the short-distance irregularities of the table's surface. If we take a ball with diameter much larger than the short-distance scale of roughness of the table and roll it over the surface, we find no evidence of the roughness, but if we use a ball whose diameter is not much larger that the scale of roughness we will see disturbances in the path of the ball resulting from its interaction with the roughness.

Similarly, a rich short-distance structure in space-time may have important effects on the propagation of particles, in a way that depends on the wavelengths of the particles. (Because of the wave-particle duality of quantum mechanics, all particles can be characterized by a wavelength, which is inversely related to their momentum.) In the tabletop analogy the diameter of the balls plays the role of the particle wavelength. For the particles we observe experimentally there should be very little wavelength dependence, but for particle wavelengths comparable with the tiny Planck length the phenomenon of particle propagation would be severely affected (imagine rolling a ball with diameter smaller than the scale of surface roughness over the table). 


\section{Hints of a new revolution?}

A key ingredient in Planck's revolutionary breakthrough was provided by Ludwig Boltzmann's earlier work, particularly his interpretation of the second law of thermodynamics as a statistical law rather than an absolute law of nature. At the time no one could have imagined that Boltzmann's work would eventually play a role in studies proving the inconsistency of classical mechanics. Is it possible that we already have some of the ingredients of a new revolution today? In recent years, several theoretical ideas have emerged that would require a change of perspective as radical as Boltzmann's. These include the theories that quantize space-time discussed above, which explain the classical properties of space-time that are perceived by long-wavelength particles as a sort of average over the richer microscopic structure of quantum spacetime. This is not unlike Boltzmann's description of the second law of thermodynamics as a statistical outcome of the properties of the many microscopic constituents of a thermodynamic system.

On the experimental side the crucial input for Planck's breakthrough came from puzzling data on the radiation emitted by hot bodies in thermal equilibrium with their surroundings. The light from these bodies is a mixture of different frequencies (colours). The classical formula linking the radiation emitted at different frequencies with the temperature of the hot body works well for radiation at low frequencies, but disagrees with experimental data at high frequencies. Planck observed that this puzzle could be solved by assuming that energy could be emitted or absorbed only in discrete amounts, and the quantum of energy was born.

Among the experimental puzzles confronting fundamental physics today there are a few that could have the potential to lead to a profound renewal of fundamental physics. A relevant illustrative example is provided by the observation of cosmic rays above the so-called Greisen-Zatsepin-Kuzmin (GZK) limit [9, 10]. Cosmic rays are particles emitted by distant active galaxies. These rays produce showers of elementary particles when they pass through the Earth's atmosphere. Before reaching us ultrahigh-energy cosmic rays travel gigantic (cosmological) distances and astronomers would expect interactions with photons in the cosmic microwave background (the faint glow left over from the big bang) to cool them down, producing a cut off at higher energies. The discovery of cosmic rays with energies above this GZK cut-off [11] apparently originating from outside our Galaxy, cannot be explained by current theories.

To calculate the GZK limit theorists have to take into account Lorentz symmetry, a property of classical space-time. It has been suggested that the observation of cosmic rays above the GZK limit could be explained by assuming very small modifications to the predictions of Lorentz symmetry [12, 13. A plausible origin for such a modification comes from an unexpected source - the quantization of space-time. Remarkably, the value of the GZK limit predicted by calculations within some of the scenarios for quantization of space-time turns out to be consistent with the astronomical data [14].

There are other plausible explanations for the cosmic-ray puzzle, but the explanation involving a quantum space-time is the only one that would simultaneously solve another similar puzzle [14, 15]. Astronomers have detected high-energy photons from distant astrophysical sources that, according to the classical picture of space-time, should not be able to reach us because they are expected to interact with the cosmic 
infrared background. This means that there should be a theoretical upper limit to the energies of the photons, similar to that for cosmic rays. Experimental evidence for such puzzles is just emerging, so it is too early to build a case for a quantum description of space-time. Nonetheless their existence is intriguing, and the mere possibility of such effects promotes the study of quantum space-times from the realm of science fiction to that of proper science.

\section{The equivalence principle.}

Experiments that can observe the interplay between general relativity and quantum mechanics are not easy to design. The tiny scale of the Planck length at which these effects are expected to emerge, and the extreme weakness of gravity compared with the other forces of nature, have led many to believe that the study of quantum gravity would be beyond the reach of doable experiments. Moreover, there appears to be a fundamental theoretical issue that affects the experimental study of gravitational forces at the quantum level. The standard approach to the operative definition of forces in quantum mechanics is in conflict with the Equivalence Principle, one of the cornerstones of general relativity.

In the study of all other forces, the operative definition of the fields involves experimental particles with two primary characteristics: their charge under the influence of the force field and their inertial mass. As shown in a celebrated paper by Bohr and Rosenfeld [16], the only way to extract accurate information on the force field is to use particles with large inertial mass and small charge. In the case of studies of gravitational fields this type of strategy would require particles with large inertial mass and small gravitational mass (which is the gravitational charge in the sense that it sets the strength of the gravitational forces felt by the particle), but the equivalence principle requires inertial and gravitational masses to be equal. Therefore the standard (BohrRosenfeld) strategy appears to lead 2, (4) to a fundamental limitation on the accuracy with which gravitational fields can be measured (and operatively defined).

\section{The experimental frontier.}

Despite these many challenges, in the last quarter of the twentieth century physicists have started to make some progress in the experimental study of quantum gravity. A first significant step was taken in the mid-1970s with experiments that, while not being capable of testing quantum properties of space-time itself, studied how the presence of strong gravitational fields affected the quantum mechanical behaviour of microscopic particles [17, 18]. These experiments established that the gravitational fields generated by the Earth affect interference experiments in a way that is completely analogous to the influence of electromagnetic fields.

The first experimental studies [19, 20, 21] with sensitivity to conjectured quantum properties of space-time were proposed in the 1980s, when it was realized that experiments testing one of the fundamental symmetries of particle physics, known as CPT, were finally reaching sensitivity levels that could plausibly detect minute modifications of these symmetries caused by the quantum properties of space-time. The sensitivity of CPT experiments (in particular studies of particles known as neutral kaons [22]) has 
continued to improve over the past few decades. So far they have provided no evidence of any quantum property of space-time.

For several years tests of CPT symmetry were the only examples of experiments being analysed from the point of view of quantum space-time, but over the past few years more proposals have been put forward. The most sensitive among these experiments are the mentioned searches for departures from Lorentz symmetry in cosmic-ray physics. Besides the GZK limit, another key implication of Lorentz symmetry - the prediction that the time needed by a massless particle to propagate over a given distance should not depend on the wavelength of the particle - is going to be tested with extremely high accuracy by astrophysics experiments now in preparation. Quantum space-time might alter the propagation of gamma-rays collected from distant gamma-ray bursts [23] some of the most powerful explosions in the Universe. Within 5 or 10 years the next generation of gamma-ray telescopes, such as the GLAST space mission [24], will reach sensitivity levels sufficient for testing the type of minute wavelength-dependence which (as illustrated by the tabletop analogy described above) could be a property of quantum space-time.

In addition to tests of Lorentz and CPT symmetries, it might even be possible to explore directly the structure of space-time itself. Modern gravity-wave interferometers are built to detect tiny fluctuations in the distances between some test masses that might be caused by a passing gravity wave 25. Gravity waves are ripples in the fabric of space-time predicted by Einstein's classical theory of gravity, but they have never been detected. In a recent paper [26], I observed that gravity-wave interferometers could also be used in attempts to detect quantum fluctuations of space-time; in fact, any physical process that induces fluctuations in the distances between the test masses will affect the interference effect. Interferometers under construction, such as the LIGO detector in the United States and the VIRGO detector in Italy, could be sensitive [26 to fluctuations at scales that correspond to the Planck length, but this possibility depends very strongly [4] on the actual mechanism of these fluctuations, and much theoretical work is still needed in order to establish which fluctuation mechanisms give rise to consistent pictures of space-time.

\section{Prospects for the 21st century.}

After 100 years of quantum theory and more than 70 years of failed attempts to unify general relativity and quantum mechanics, we finally have some promising theoretical and experimental avenues for the investigation of the interplay between relativity and quantum theory. There is a strong theoretical desire to unite these two theories, but without a healthy experimental programme our best models would remain as mere speculation. For now, the experiments are limited in number and their sensitivities are still only at levels that would correspond to rather optimistic estimates of the effects (estimates that rely on the assumption that the strength of some of the effects induced by the unification of general relativity and quantum mechanics will only be mildly suppressed by the smallness of the Planck length). Nonetheless, it seems reasonable to expect that a growing number of experimental and theoretical ideas will emerge over the 21st century and will eventually allow us to bring clarity into the challenge to quantum mechanics presented by the need to come to terms with general relativity. 


\section{Acknowledgements}

The writeup of this article went through several rounds of editing. I felt I should use this opportunity to convey to scientists from other fields an exciting picture of frontier research within and beyond quantum mechanics, but some aspects of the subject are very technical and I feared that the final result might be disappointing in all respects: in spite of painful oversimplifications and painful compromizes on factual accuracy I could have missed the target of a piece readable by non-experts. In the end a draft that I could live with did mature, but only thanks to several rounds of feed-back and precious guidance from John Stachel and Sarah Tomlin. The generous contribution of John and Sarah is very greatfully acknowledged.

\section{References}

[1] A. Zeilinger, Nature 408 (2000) 639-641.

[2] J. Stachel, Early history of Quantum Gravity, in "Black Holes, Gravitation Radiation and the Universe" (Kluwer Academic, Dordrecht, 1999).

[3] C. Rovelli, The century of the incomplete revolution, J. Math. Phys. 41 (2000) 3776-3800.

[4] G. Amelino-Camelia, Are we at the dawn of quantum-gravity phenomenology?, grqc/9910089, notes based on lectures given at the XXXV Karpacz Winter School of Theoretical Physics From Cosmology to Quantum Gravity, Polanica, Poland, 2-12 February, 1999 (published in the volume entitled "Towards Quantum Gravity", Springer-Verlag Heidelberg 2000, edited by J. Kowalski-Glikman).

[5] G. 't Hooft, Quantum gravity as a dissipative deterministic system, Class. Quantum Grav. 16 (1999) 3263-3279.

[6] A. Ashtekar, C. Rovelli and L. Smolin, Weaving a classical geometry with quantum threads, Phys. Rev. Lett. 69 (1992) 237-240.

[7] M.B. Green, J.H. Schwarz and E. Witten, Superstring theory (Cambridge Univ. Press, Cambridge, 1987).

[8] J. Polchinski, String theory (Cambridge Univ. Press, Cambridge, 1998).

[9] K. Greisen, Phys. Rev. Lett. 16 (1966) 748-751.

[10] G. T. Zatsepin and V. A. Kuzmin, Sov. Phys.-JETP Lett. 4 (1966) 78-80. 
[11] D. J. Bird et al., Astrophys. J. 441 (1995) 144-151.

[12] S. Coleman, S.L. Glashow, High-energy tests of Lorentz invariance, Phys. Rev. D59 (1999) 116008.

[13] T. Kifune, Invariance violation extends the cosmic ray horizon?, astro-ph/9904164, Astrophys. J. Lett. 518 (1999) L21-L24.

[14] G. Amelino-Camelia and T. Piran, astro-ph/0008107.

[15] H. Muir, Blazars, New Sci. (23 September 2000) 32-35.

[16] N. Bohr and L. Rosenfeld, Zur frage der messbarkeit der elktromagnetischen feldgroessen, Kgl. Danske Videnskab S. Nat. Fys. Medd. 12 (1933) 1; english translation in Selected papers of Leon Rosenfeld, p.357-400 (Reidel, Dordrecht/Boston/London, 1979)

[17] R. Colella, A.W. Overhauser and S.A. Werner, Observation of gravitationally induced quantum interference, Phys. Rev. Lett. 34 (1975) 1472-1474.

[18] D.V. Ahluwalia, Quantum gravity: testing time for theories, gr-qc/9903074, Nature 398 (1999) 199.

[19] J. Ellis, J.S. Hagelin, D.V. Nanopoulos and M. Srednicki, Search for violations of quantum mechanics, Nucl. Phys. B241 (1984) 381-405.

[20] V.A. Kostelecky and R. Potting, Phys. Rev. D51 (1995) 3923.

[21] P. Huet and M.E. Peskin, Violation of CPT and quantum mechanics in the $K_{0}-\bar{K}_{0}$ system, Nucl. Phys. B434 (1995) 3-38.

[22] J. Ellis, J. Lopez, N.E. Mavromatos, D.V. Nanopoulos and CPLEAR Collaboration, Test of CPT Symmetry and Quantum Mechanics with Experimental data from CPLEAR, Phys. Lett. B364 (1995) 239-245.

[23] G. Amelino-Camelia, J. Ellis, N.E. Mavromatos, D.V. Nanopoulos and S. Sarkar, Tests of quantum gravity from observations of $\gamma$-ray bursts, astro-ph/9712103, Nature 393 (1998) 763-765.

[24] A. de Angelis, astro-ph/0009271.

[25] P.R. Saulson, Fundamentals of interferometric gravitational wave detectors (World Scientific, Singapore, 1994).

[26] G. Amelino-Camelia, Gravity-wave interferometers as quantum-gravity detectors, gr-qc/9808029, Nature 398 (1999) 216-218. 